\title{
Effect of pneumatic compression therapy on lymph movement in lymphedema-affected extremities, as assessed by near-infrared fluorescence lymphatic imaging
}

\author{
Melissa B. Aldrich ${ }^{* \S}$, Deborah Gross ${ }^{\dagger}$, John Rodney Morrow*, \\ Caroline E. Fife ${ }^{*}$ and John C. Rasmussen* \\ *Center for Molecular Imaging \\ The Brown Institute for Molecular Medicine \\ UTHealth, Houston, Texas, USA \\ ${ }^{\dagger}$ Mego Afek/Lympha Press USA \\ Freehold, New Jersey, USA \\ ¥CHI St. Luke’s Health \\ The Woodlands Hospital \\ The Woodlands, Texas, USA \\ $\S_{\text {melissa.b.aldrich@uth.tmc.edu }}$
}

Received 12 May 2016

Accepted 23 August 2016

Published 28 September 2016

\begin{abstract}
Previous studies have shown cost effectiveness and quality-of-life benefit of pneumatic compression therapy (PCT) for lymphedema (LE). Insurers, such as the Centers for Medicare/Medicaid (CMS), however, desire visual proof that PCT moves lymph. Near-infrared fluorescence lymphatic imaging (NIRFLI) was used to visualize lymphatic anatomy and function in four subjects with primary and cancer treatment-related LE of the lower extremities before, during, and after PCT. Optically transparent and windowed PCT garments allowed visualization of lymph movement during single, $1 \mathrm{~h}$ PCT treatment sessions. Visualization revealed significant extravascular and lymphatic vascular movement of intradermally injected dye in all subjects. In one subject with sufficient patent lymphatic vessels to allow quantification of lymph pumping velocities and frequencies, these values were significantly increased during and after PCT as compared to pre-treatment values. Lymphatic contractile activity in patent lymphatic vessels occurred in concert with the sequential cycling of PCT. Direct visualization revealed increased lymphatic function, during and after PCT therapy, in all LE-affected extremities. Further studies are warranted to assess the effects of PCT pressure and sequences on lymph uptake and movement.
\end{abstract}

Keywords: Lymphedema; pneumatic compression therapy; near-infrared fluorescence.

$\S_{\text {Corresponding author. }}$

This is an Open Access article published by World Scientific Publishing Company. It is distributed under the terms of the Creative Commons Attribution 4.0 (CC-BY) License. Further distribution of this work is permitted, provided the original work is properly cited. 


\section{Introduction}

Lymphedema (LE) is an accumulation of excessive lymph fluid in the subcutaneous tissues, due to inadequate transport capacity of the lymphatic system. The inadequate transport can result from impairment of the lymphatic vessels, as occurs in up to $30 \%$ of U.S. cancer survivors, due to lymph vessel disruption, ${ }^{1-4}$ from lymph vessel malformations, as in genetically associated (primary) LE, ${ }^{5,6}$ from chronic venous insufficiency, ${ }^{7}$ and other causes.

LE disposes the affected area to an ongoing inflammatory process that, if untreated, can progress to skin fibrosis, adipose tissue accumulation, and further accumulation of fluid, causing disfiguring swelling, disability, and infections such as cellulitis. Studies have shown that if LE is recognized early, and treatment begun at initial stages, permanent swelling due to adipose tissue accumulation, as well as fibrotic skin changes and cellulitis, can often be avoided ${ }^{8}$ Patients in the US first use "conventional" treatment (compression with or without manual lymphatic drainage, or MLD) for months. In some areas of the US, pneumatic compression devices may be covered by insurers, but the patient must first fail using a basic PCT pump before being allowed to try an advanced pump. Lymphaticovenous anastomoses and lymph node transplant surgeries have been performed on selected LE patients, and have demonstrated decreased cellulitis incidence and limb swelling in many cases. ${ }^{9-14}$ Compression garment use after the surgeries is still required to maintain limb volume reductions in most cases to prevent further lymph and subcutaneous adipose accumulation. Surgical treatment is indicated for patients who do not improve with conservative measures, or for patients in whom the extremity is so large that it impairs daily activities and prevents successful conservative management, ${ }^{9}$ but US medical insurers do not routinely cover the surgeries.

Conservative treatment for LE consists of massage and compression. Clinic-based treatment, known as complete decongestive therapy (CDT), consists of multilayered compression bandaging, exercise, skin care, education, and therapeutic, directional massage (MLD). ${ }^{15}$ MLD is applied with the goal of stimulating lymphatic uptake in the affected area, or in the cases where there is a blockage or obliteration/dysfunction of local lymphatics, rerouting the fluid to areas with functioning lymphatics. MLD is applied in specific patterns, and has been documented to increase lymphatic uptake and propulsion. ${ }^{16}$ Immediately after MLD, compression bandaging is applied, which functions by raising the hydrostatic pressure in subcutaneous tissues, enabling movement of fluid through open channels and improving conditions for uptake of fluid into the lymphatics. MLD and lymphatic compression bandaging are skilled techniques, and are administered by a trained therapist, requiring repeated and numerous clinic visits.

Pneumatic compression therapy (PCTs) devices are an alternate or adjunctive method of LE therapy. PCTs are used in-clinic as part of CDT treatment, as a home-based continuation of CDT, or as an alternative therapy. PCT systems consist of a pump that inflates a garment worn over the affected area, in an inflation pattern of duration and pressure that varies by PCT construction and manufacture. These systems are generally easy to operate by the patient in the home environment, in accordance with the prescription of a licensed medical practitioner. ${ }^{17,18}$

Previous studies have shown PCTs provide significant edema reduction and long-term maintenance, ${ }^{19,20}$ can provide treatment equivalent in edema reduction outcome to in-clinic methods, ${ }^{21}$ can maintain edema reduction after discharge from in-clinic sessions, ${ }^{22}$ and can reduce the incidence of cellulitis infection. ${ }^{23}$ Therefore, PCT use could potentially make a significant difference in the level of care for LE patients who do not have access to clinics with trained therapists, and could provide favorable treatment outcomes. However, adoption of these systems by clinicians has been limited, because visible evidence of the efficacy of PCT to stimulate lymph uptake, propulsion, and movement, including transport of proteins in lymph fluid, has been deemed lacking or inconclusive, ${ }^{24}$ and by opinion in the literature that PCTs do not stimulate lymphatic transport. ${ }^{25}$ These claims persist, despite lymphoscintigraphy studies demonstrating tracer uptake by the lymphatics during PCT treatment. ${ }^{26}$

Near-infrared fluorescence lymphatic imaging (NIRFLI) technology uses a sensitive camera and optics system to detect fluorescence emitted from a fluorescent dye (indocyanine green, or ICG) that, once injected intradermally, binds to albumin and other proteins that are taken up into the lymph. ${ }^{27,28}$ 
Dim near-infrared laser illumination of skin allows tissue-penetrating light to excite the dye, and the resulting fluorescent photons are collected to produce images of lymphatic vessel architecture and movies displaying movement of lymph as it is "pumped" from distal limb areas. ${ }^{29,30}$ This study utilized NIRFLI technology to determine influence of PCT treatment on lymph fluid movement and lymphatic uptake in LE-affected extremities.

The PCT utilized in this investigation, Lympha Press Optimal ${ }^{\circledR}$ (Mego Afek Ac, Ltd., Israel $)^{18}$ consists of a pump that applies pressure to multiple individual chambers inside the garment. The chambers, which overlap each other in a manner similar to compression bandaging, inflate in sequence from distal to proximal, hold briefly, and then deflate for a brief intermission before resuming the distal-to-proximal cycle. Prior to engaging the sequential compression, the PCT applies a peristaltic massage cycle to the proximal areas. Treatment pressures are selectable according to clinician assessment $^{17}$ from $20 \mathrm{mmHg}$ to $90 \mathrm{mmHg}$.

Herein, we provide images and movies of lymphatics, obtained using NIRFLI technology, before, during, and after PCT, in a study cohort of four subjects with different presentations of LE. The images of lymphatics of the four subjects obtained through clear and windowed PCT garments enabled real-time visualization of lymph movement in response to $\mathrm{PCT}$. Images and movies from these patients demonstrate the ability of PCT to move fluids proximally in patent lymphatic vessels and through extravascular spaces. Comparison of images from the first subject, who serendipitously was imaged 18 months prior without any PCT treatment, provides convincing evidence that enhanced proximal lymph movement results from PCT. Notably, subject 3 had sufficient patent lymphatic vessels that allowed quantification of lymphatic function before, during, and after PCT.

\section{Methods}

\subsection{Human subjects}

The grade/type of LE, previous LE treatment, garments used, and other details of the study subjects are listed in Table 1. A total of four subjects were recruited, and all were enrolled. Written consent was obtained from all subjects before imaging and PCT, and all studies were conducted under institutional IRB and FDA approval (IND 102, 765).

\subsection{NIRFLI imaging system and PCT system}

The NIRFLI imaging system has been described previously and is illustrated in Visualization $1 .{ }^{27,29}$ The custom-built, investigational device is equipped with a NIR-sensitive, military grade image intensifier and a 16-bit, frame transfer, charge coupled device (CCD) camera. Images were acquired by illuminating the tissue with the diffuse output $\left(\leq 1.9 \mathrm{~mW} / \mathrm{cm}^{2}\right)$ of a $785 \mathrm{~nm}$ laser diode and collecting the resulting $830 \mathrm{~nm}$ fluorescent signal emanating from the tissue. ${ }^{30}$ The PCT system used consisted of the Lympha Press Optimal ${ }^{\circledR}$ programmable pump and appliance garments. Garment 1 consisted of multi-chambered pants with clear windows at dorsal foot/ankle, medial knee, and naval areas. Garment 2 consisted of a multichambered leg garment made entirely of clear material. With the exception of the external materials utilized for optical visualization, the garments were not altered from their standard, marketed form. In some cases, two identical garments were used (one garment on each leg). The garments are pictured later.

\subsection{ICG injection, PCT treatment, NIRFLI, and data analysis}

Immediately prior to imaging, each subject received 12-14 intradermal injections, each containing 25 micrograms ICG in $0.1 \mathrm{cc}$ saline, for a 300-350 micrograms total dose, as illustrated in Fig. 1. Injection sites, per side, included two in dorsal foot, one in medial ankle, one in medial and one in lateral calf, and one in thigh. Subjects 1 and 2 received two additional injections in the peri-navel area.

Imaging began immediately after ICG injection. After initially imaging for $30 \mathrm{~min}$, the PCT garment(s) was donned for approximately $1 \mathrm{~h}$ of treatment (Fig. 2).

NIRFLI was conducted during PCT treatment, after which the PCT garment was removed and imaging was performed for an additional $30 \mathrm{~min}$. PCT consisted of approximately 60 cycles of 45,40 , 35 , and $30 \mathrm{mmHg}$ pressure applied in a distal-toproximal gradient for $50 \mathrm{~s}$ per cycle. Using garment 


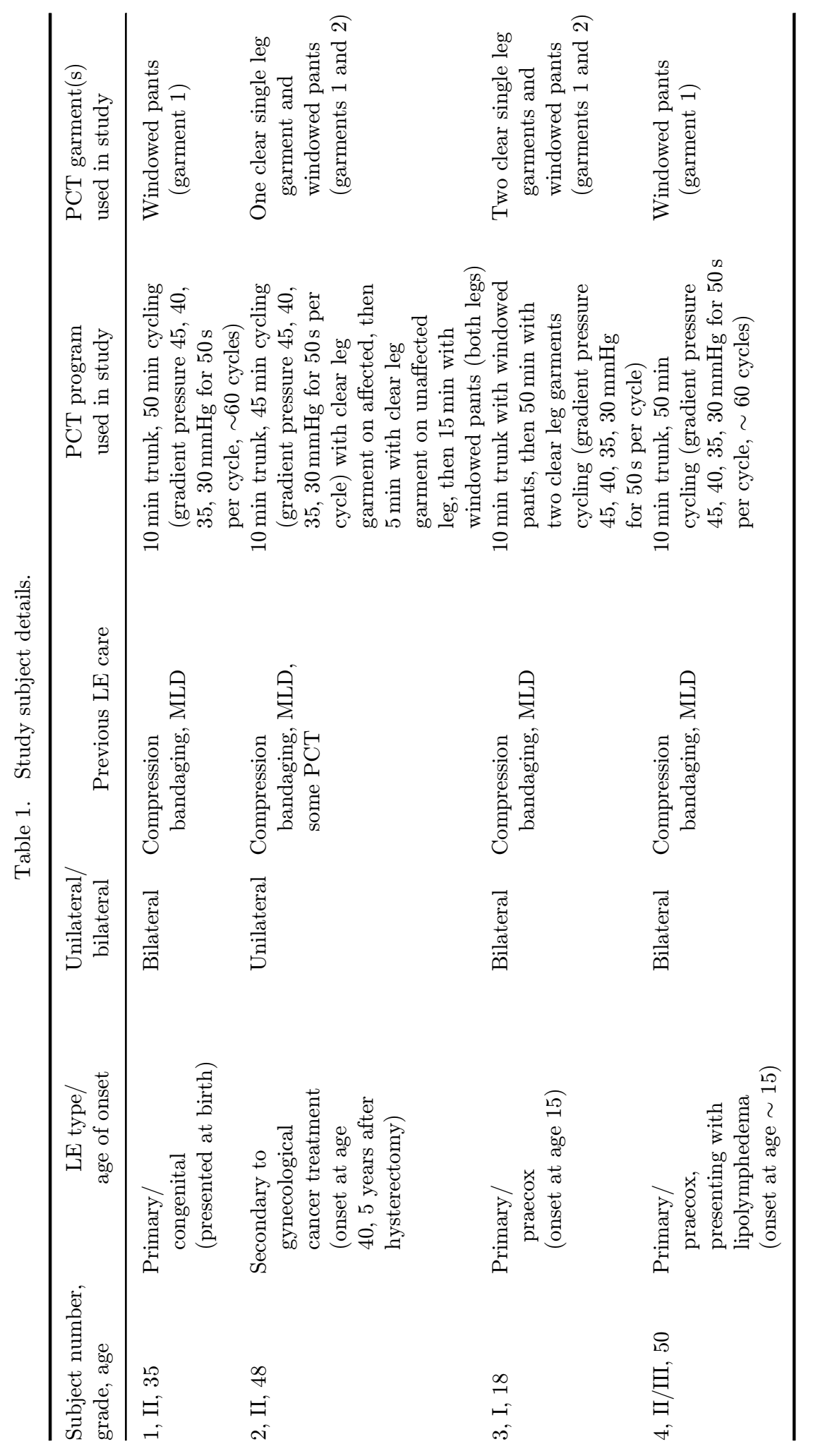




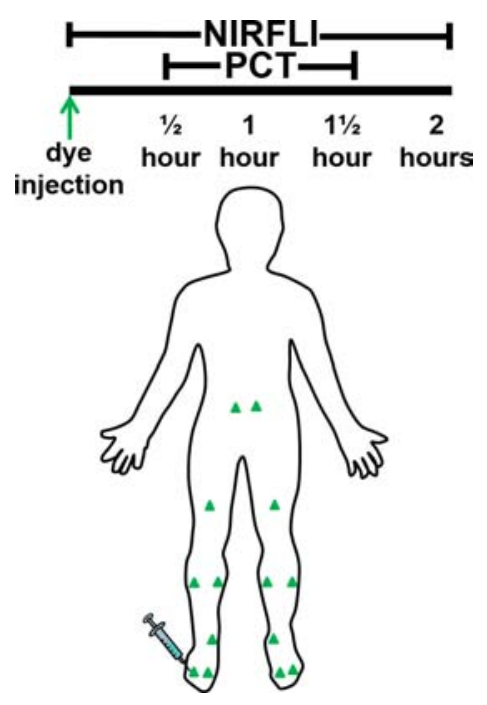

Fig. 1. (Color online) Study logistics. Green triangles indicate ICG injection sites. NIRFLI was conducted for 30 min before donning of Lympha Press PCT garment. NIRFLI was continued during PCT, which consisted of continuous cycles of 45, 40, 35, and $30 \mathrm{~mm} \mathrm{Hg}$ for $1 \mathrm{~h}$, starting at trunk, and then moving from lower to upper legs, and, in the case of the pants, the abdomen. PCT garment removal was followed by 30 min of imaging.
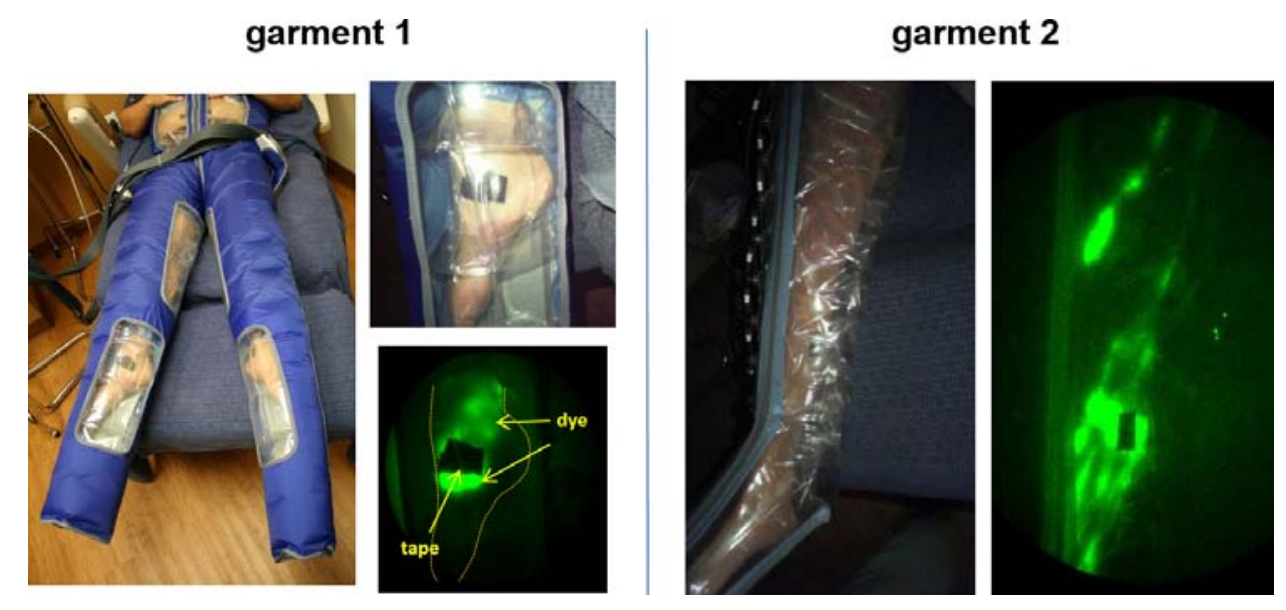

Fig. 2. Study PCT garments and NIRFLI images obtained with garments. Garment 1 (pants) featured clear windows at foot, medial knee, and anterior abdominal areas. Garment 2 (one leg) was made entirely of clear material. Extravascular, as well as vascular lymph in linear and tortuous vessels are readily visible using these garments.

1 (pants), the trunk area was drained first, then distal (foot) areas, lower leg, and finally upper leg areas. Trunk drainage with garment 1 (10 min) preceded use of completely clear garment 2 , which drained foot, leg, and then upper leg areas. Images were analyzed and compiled into movies using ImageJ software (NIH) as described previously. ${ }^{27,29,31,32}$ Mean values for lymphatic pulsatile velocities and periods (time between pulses) were compared using Student's $t$-tests (Microsoft Excel). Of note, during NIRFLI, dye/lymph migration to terminal destinations is typically evident within
$15 \mathrm{~min}$ of ICG injection. Pre-treatment NIRFLI images shown in the figures were taken 15 min after dye injection, and post-treatment NIRFLI images were taken $1-1 / 2 \mathrm{~h}$ after dye injection.

\section{Results}

\subsection{Lymph moved in response to PCT (Subject 1)}

Comparison of NIRFLI images from the two imaging sessions of subject 1 provided evidence of PCT 


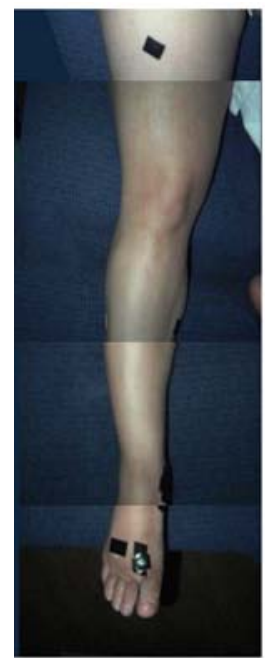

white light

image

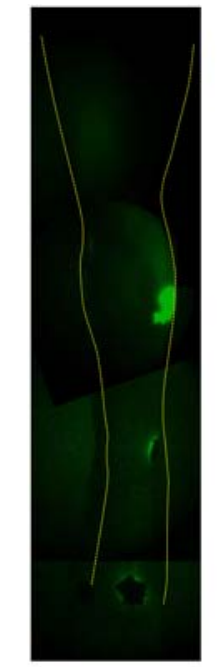

1-1/2 hours after dye injection, no PCT

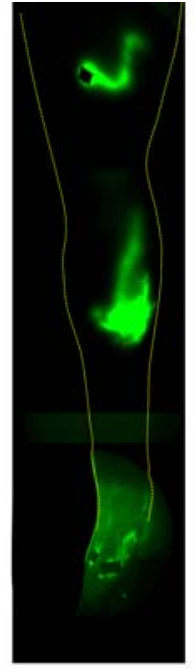

before PCT

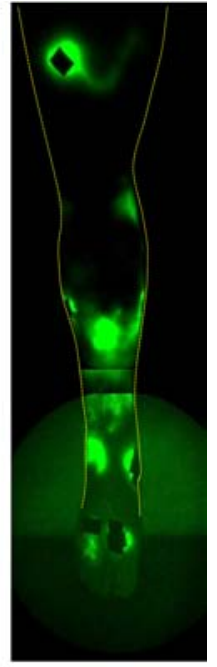

after PCT

Note: Full-leg images are montages of smaller regional images.

Fig. 3. Fluorescent dye/lymph distribution in right anterior leg before and after PCT in subject 1. (leftmost) White light image, (second from left) NIRFLI image 1-1/2-2 h after dye injection, from first imaging session, in which no PCT was performed (18 months prior to second imaging session), (third from left) NIRFLI image before PCT in second imaging session, (rightmost) NIRFLI image after PCT in second session (1-1/2-2 h after dye injection).

efficacy, as shown by more complete filling of lymph vessels with ICG during the PCT session as compared to a NIRFLI session of the same duration that did not include PCT. NIRFLI images of the right anterior leg of subject 1, shown in Figs. 3 and 4, provide visual evidence that ICG-laden lymph moved proximally from the injection sites $90-135$ min after injection in both the first and second imaging sessions.

In the first imaging session, with no PCT, 90$120 \mathrm{~min}$ after injection, little dye was observed away from injection sites; in fact, only a small area of diffuse/extravascular dye around the medial shin

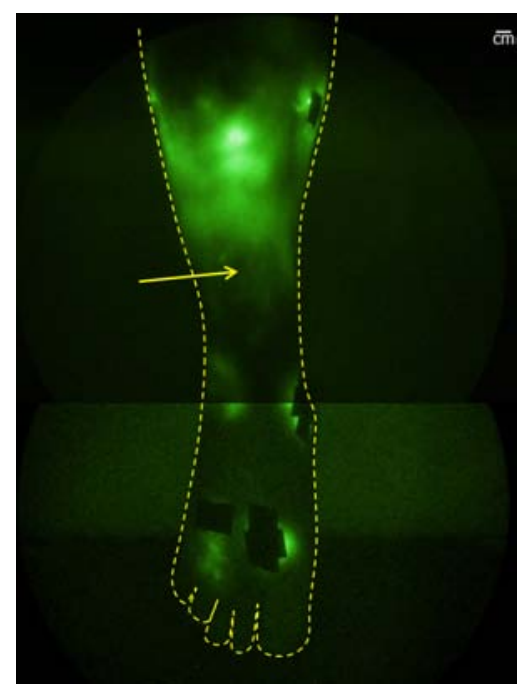

Note: Image shown is montage of smaller regional images. Scale bar $=1 \mathrm{~cm}$.

Fig. 4. NIRFLI image of subject 1's lower right anterior leg, after PCT treatment. Extravascular/diffuse, as well as primary vessel vascular/linear (small vessels noted by arrow) dye/lymph is visible. Injection sites are covered by small round bandages or small rectangles of black tape to prevent camera saturation. 


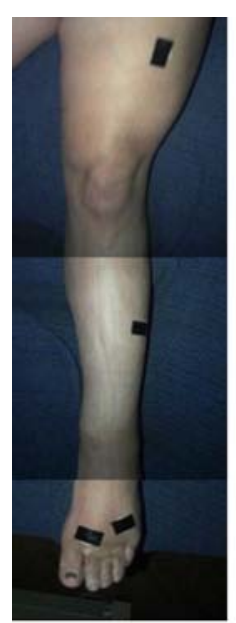

white light image

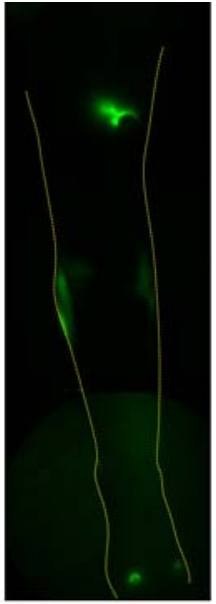

1-1/2 hours after dye injection, no PCT

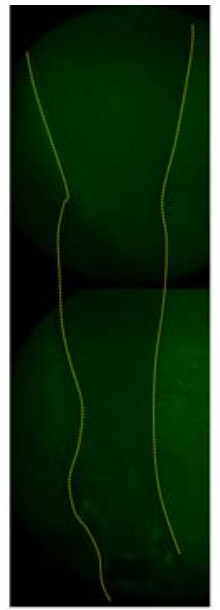

before PCT

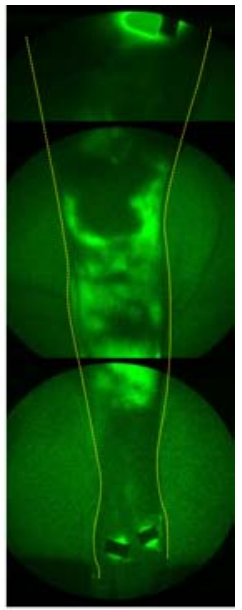

after PCT

Note: Full-leg images are montages of smaller regional images.

Fig. 5. Fluorescent dye/lymph distribution in left posterior leg before and after PCT in subject 1. (leftmost) white light image, (second from left) NIRFLI image 1-1/2-2 h after dye injection, from first NIRFLI imaging session, in which no PCT was performed, 18 months prior to second imaging session, (third from left) NIRFLI image before PCT in second imaging session, (rightmost) NIRFLI image after PCT in second session (1-1/2-2 hours after dye injection).

injection site was visible (Fig. 3, second image from left). In the second imaging session, before PCT, only areas near the foot, medial calf, and thigh injection sites displayed fluorescent dye (Fig. 3, third image from left). After PCT, large areas of ICG/ lymph were visible in the right anterior shin (Fig. 3, rightmost image). Dye was visualized proximal to injection sites, potentially from flow through interstitial channels that could empty into lymphatic collectors. Closer inspection of the right anterior shin area in
Fig. 4 reveals plenteous extravascular/diffuse with some linear/vascular ICG-laden lymph apparent (seen as fine primary lymphatic vessels just above ankle).

Small areas of extravascular/diffuse ICG-laden lymph protruding from dorsal foot and medial ankle injection sites are also evident, as is a large extravascular/diffuse area at the lateral ankle. Images of the left anterior leg of subject 1 , shown in Figs. 5 and 6, likewise reveal more extravascular/ diffuse and vascular/linear ICG-laden proximal

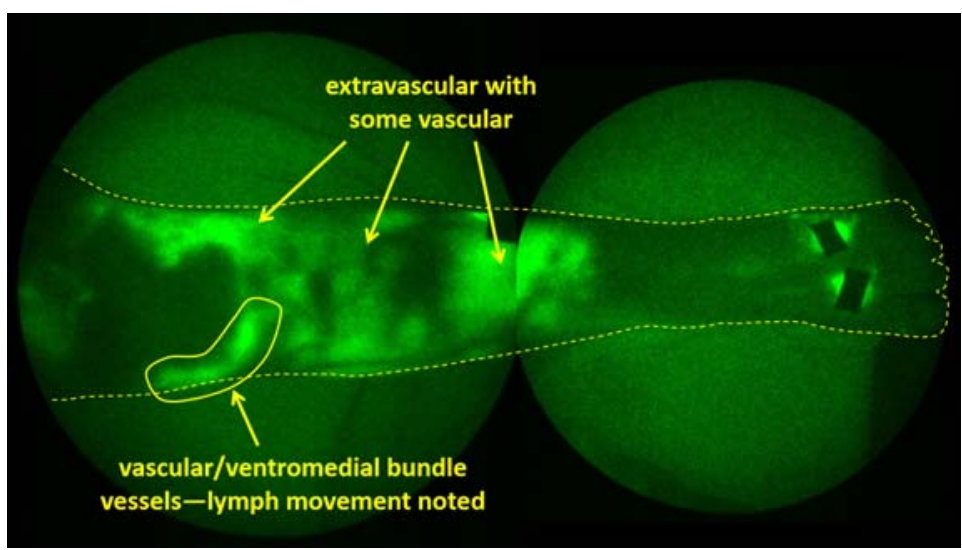

Note: Image shown is montage of smaller regional images.

Fig. 6. NIRFLI image of subject 1's lower left anterior leg, after PCT treatment. Extravascular/diffuse and vascular/linear dye/ lymph is visible. Ventromedial lymphatic bundle vessel is evident, and pumping through this vessel was noted. 
lymph movement from injection sites after PCT, as compared to the prior session without PCT.

Without PCT, NIRFLI images taken 90-135 min after injection show limited movement of ICG on the dorsal foot and shin injection sites (Fig. 5, second image from left), and a small vessel in the thigh which carries ICG-laden lymph to the inguinal area.

In the second imaging session, following PCT, abundant extravascular/diffuse and vascular/linear ICG-laden lymph is clearly visible (Fig. 5, rightmost image), compared to before PCT (Fig. 5, third image from left, and Fig. 6), including vascular/linear lymph in ventromedial bundle vessels (below kneecap), as well as in the dorsal foot (shown as straight, linear vessels emerging from dorsal foot injection sites). In addition to lymph pumping through the ventromedial bundle (knee

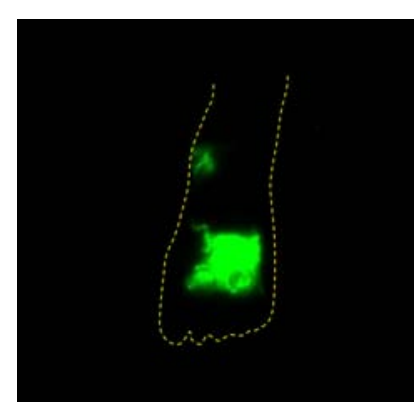

(a) area, Visualization 2, video), extravascular/diffuse and vascular/linear ICG-laden lymph is apparent in left anterior shin, lateral knee, and lower thigh areas.

\subsection{Lymph movement during PCT ( Subject 2)}

The use of a completely clear PCT garment (garment 2) allowed greater real-time visualization of ICG-laden lymph during the imaging session of subject 2, with unilateral acquired LE.

The left/affected dorsal and medial foot displayed primarily extravascular/diffuse ICG $15 \mathrm{~min}$ after ICG injection, but after PCT, most of the ICG-laden lymph is located within lymphatic vessels in the foot (Fig. 7).

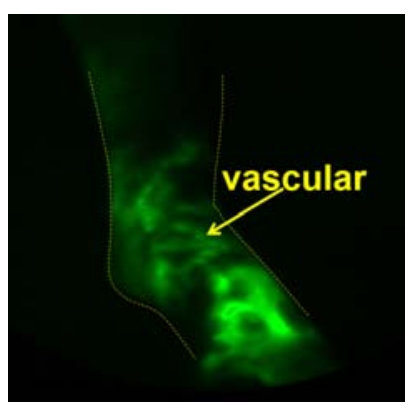

(b)

Fig. 7. NIRFLI image of subject 2's anterior medial left foot before (a) and after (b) PCT treatment in subject 2. Extravascular/ diffuse and vascular/linear (arrow) dye/lymph are visible.

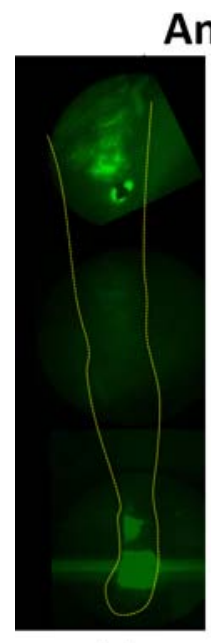

(a)
Anterior

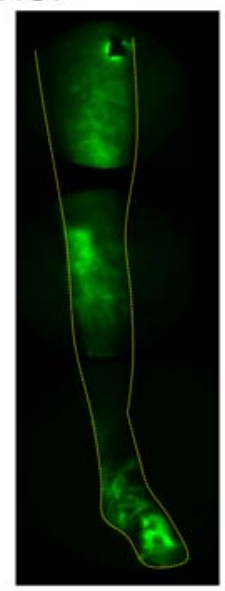

(b)

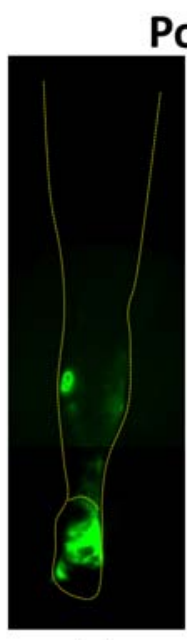

(c)
Posterior

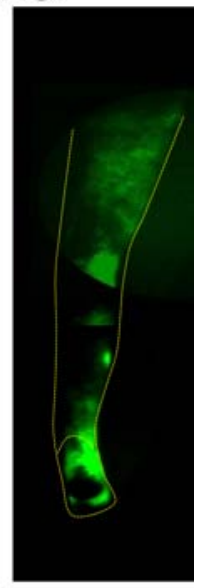

(d)

Note: Full-leg images are montages of smaller regional images.

Fig. 8. Fluorescent dye/lymph distribution in subject 2's left (LE-affected) leg before and after PCT. (a) NIRFLI image of anterior left leg before PCT, (b) anterior left leg after PCT, (c) posterior left leg before PCT, (d) posterior left leg after PCT. 
Figure 8 shows a montage anterior leg view before PCT (Fig. 8(a), and Fig. 8(b) shows this view after PCT. Figures 8(c) and 8(d) show before and after PCT images of the posterior leg. ICG-laden lymph movement to knee and thigh areas after PCT is notable. The right/unaffected leg did not show any differences in lymph distribution before (Visualizations 3a and 3c) and after (Visualizations $3 \mathrm{~b}$ and $3 \mathrm{~d})$ PCT.

\subsection{Lymph pumping through patent lymphatic vessels is improved dur- ing PCT and after PCT (Subject 3)}

Despite skin thickening and extravascular ICG evident at both feet/ankles, subject 3 (grade I/II praecox) displayed working lymphatic vessels.

Figure 9 shows that PCT recruited the use of femoral/thigh lymphatic vessels. The use of two
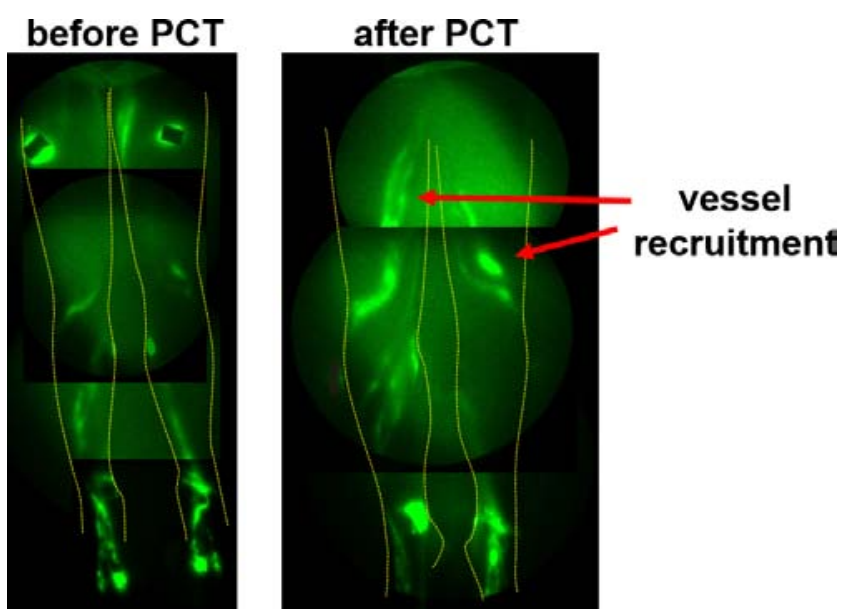

Note: Thigh vessel recruitment with PCT (arrows)

Fig. 9. Subject 3's anterior NIRFLI images before and after PCT.
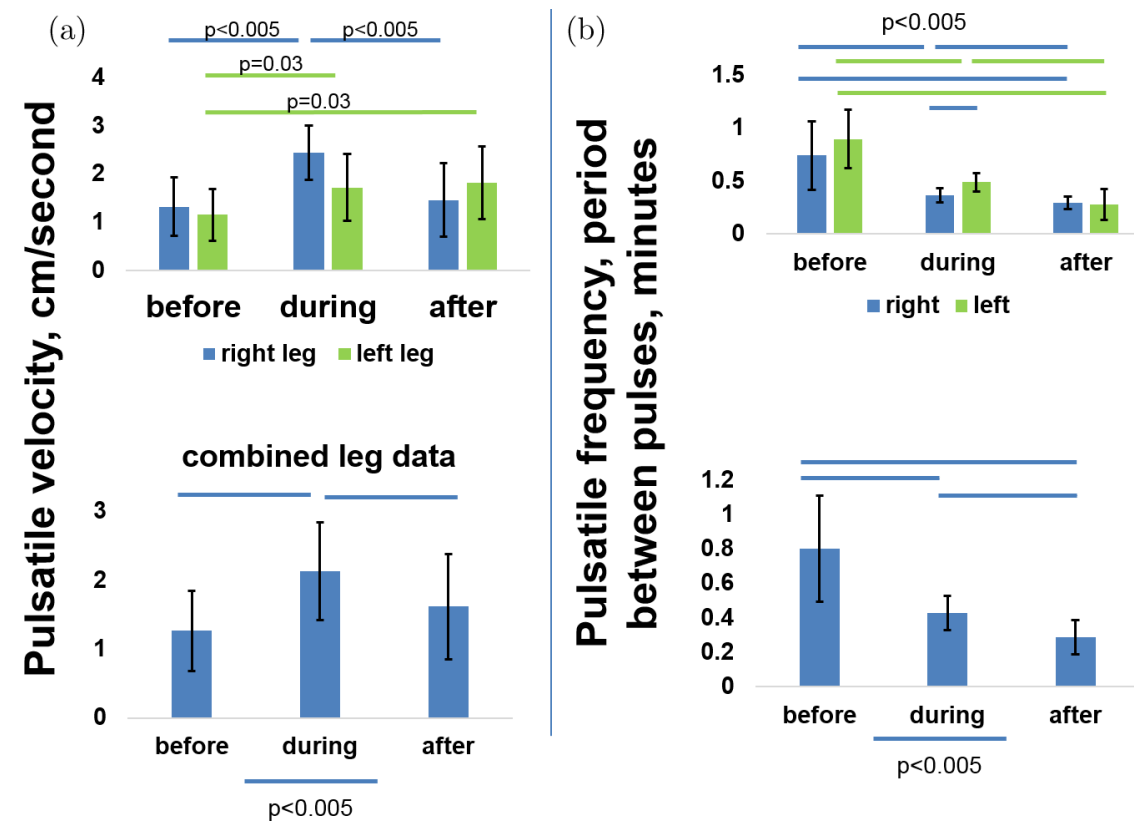

Fig. 10. Pulsatile velocities and frequencies increased with PCT in subject 3. (a) Lymphatic vessel pulsatile velocities (centimeters/ minute) and frequencies (expressed in periods, or minutes between pulses) in left and right legs before, during, and after PCT, (b) data from both legs combined. Bars $=p<0.005$, unless otherwise indicated. 
completely clear leg garments during PCT allowed quantification of lymphatic pumping velocities and frequencies before, during, and after PCT (Fig. 10).

The histogram in Fig. 10(a) shows calculated pulsatile velocities for each leg before, during, and after PCT for this bilateral subject, and Fig. 10(b) shows the same data for both legs combined. Lymph vessel pulsatile velocity increased markedly during PCT, and remained elevated above pre-PCT levels during the $30 \mathrm{~min}$ period immediately following removal of the PCT garments. Likewise, pulsatile frequency (expressed as period, or time between pulses) improved (decreased) during PCT, and stayed lower during the $30 \mathrm{~min}$ post-PCT period. Visualizations 4-6 (videos) show pulsing in the same two ventromedial bundle vessels in the right leg before, during, respectively, and after PCT (all videos are set to identical frame/second settings to allow visual comparison of pumping velocities).

\subsection{PCT increased vascular/linear lymph pumping even with adipose tissue accumulation (subject 4)}

Visualization 7 shows "before" and "after" images of subject 4's (lipolymphedema) feet and lower legs. While the overall superficial movement of ICG appears to be less than seen in other subjects, total leg volume markedly decreased after PCT $(2.2 \%$, the average for all subjects), and the videos taken during PCT reveal lymphatic contractile activity occurring in concert with the sequential cycling of PCT (Visualizations 8 [NIRFLI video] and 9 [corresponding white light video]).

\section{Discussion}

NIRFLI allows noninvasive imaging of lymphatic vasculature and nodes to depths of $3-4 \mathrm{~cm}$, evidenced by pathological confirmation in a previous study. ${ }^{32}$ Other NIRFLI studies have revealed a wide range of lymphatic vessel architecture/phenotypes in subjects with diseased lymphatics. ${ }^{28}$ Of note, the first two subjects in the present study were imaged in previous studies, and their visible lymphatic vessel architecture appeared to be relatively unchanged, after 18 and 60 months (for subject 1 and subject 2 , respectively). This finding suggests that functional lymphatics are stable and NIRFLI is reproducible, providing reliable longitudinal information on the status of functional lymphatics.

By comparing subject 1's NIRFLI images obtained 90-135 minutes post-ICG injection with and without PCT, there is evidence that lymph movement results from PCT, not the passive transit of ICG. These single-patient results indicate that $\mathrm{PCT}$ is responsible for the movement of lymph into and through lymphatic vessels and interstitial spaces and channels. In the case of this grade II subject, the movement of ICG-laden lymph was too slow to be obvious in a video format, but is readily noticeable in the pre- and post-treatment images. NIRFLI of Subject 2 using the clear unilateral PCT garment yielded real-time images of ICG movement in her entire affected leg during PCT. ICG moved proximally from foot injection sites to foot lymphatic vasculature during PCT. Subject 3, who presented with patent lymphatic vessels as shown by NIRFLI, displayed significantly increased pulsatile velocities and frequencies during and after PCT, and, in Subject 4, lymph pumping during PCT coordinated with pneumatic compression cycles. This direct response to the pressure cycle is notable, as lymph pumping is effected by opening and closing of one-way valves inside the lymph vessels, which respond to changes in hydraulic pressure with increased activity. ${ }^{33}$

Proximal-to-distal lymph flow has been observed in previous human ${ }^{34}$ and mouse inflammation ${ }^{35}$ NIRFLI studies. In the mouse study, intravital imaging revealed lymphatic vessel dilation, suggesting that lymph valve tips that do not "touch" can allow backwards lymph flow. Several mouse knockout strains with mutations in human LE-associated genes exhibit lymphatic valve malformation or failure. ${ }^{36,37}$ Indeed, our recent study found a LEassociated, inactivating mutation in a gene important to lymphatic valve cell polarity and connections, CELSR1 (Cadherin EGF LAG Seven-Pass G-Type Receptor 1), in a human family. ${ }^{38}$ It is possible that proximal-to-distal lymph flow could occur with PCT in LE patients with valve-affecting mutations, if sequential compression is inadequate to keep lymph moving in distal-to-proximal directions. Most LE patients in the US encounter LE after cancer treatment, however, and because cancer does not typically metastasize distally from affected lymph nodes into extremities, PCT is unlikely to contribute to cancer spread.

Previous studies of MLD with NIRFLI showed real-time stimulation of lymphatic propulsion by 
manual massage, in both normal controls and in LE-affected individuals. ${ }^{16}$ A previous study using NIRFLI technology with a different PCT operating with mild, variable pressure on normal control subjects and normal and affected arms of subjects with breast cancer-related LE demonstrated PCTmediated increases of lymphatic vessel fluid transport in the normal control subjects and in the unaffected arms of the LE-affected study subjects; however, the study failed to demonstrate lymphatic vascular/linear fluid movement in the LE-affected arms of the subjects tested, showing extravascular/ diffuse movement only. ${ }^{39}$

In the current study, with the sequential Lympha Press Optimal ${ }^{\circledR}$ PCT device at $45-30 \mathrm{mmHg}$, improved lymph movement during and after PCT was demonstrated in all of the affected legs of the subjects tested, with visualization of vascular/linear lymphatic propulsion and extravascular/diffuse proximal movement in all affected extremities. The synchronization of the lymphatics in time with the cycling of the device in Subject 4 indicates a direct effect of the PCT device action on the propulsion of lymph within the lymphatic vessel, while in Subject 3, the increases in pulsatility could be quantified.

As stated previously, there has been a persistent attitude among manual therapists that PCTs do not engage the lymphatic system and simply "move fluid around" or "drive fluid into the veins." In this study, NIRFLI visualization showed PCT-mediated increase in lymphatic function (when patent lymphatic vessels were present) and movement of protein-bound ICG, as well as decongestion of edema fluid through the lymphatics, in LE-affected extremities.

Other groups have used NIRF technology to investigate $\mathrm{LE},{ }^{40-42}$ and have presented fluorescent image patterns (linear, splash, stardust, and diffuse) that associate with the progression of LE severity. We have observed similar, heterogeneous NIRF image patterns in patients, albeit in possibly different progression with disease severity, and not always occurring in a proximal-to-distal progression. For example, in this study we imaged Subject 3 , in whom only ankles were affected. In this subject, the diffuse pattern was noted only at ankles, with linear patterns in feet, knee, and thigh areas. The diffuse dermal backflow pattern did not originate at the most proximal areas of the limbs, in contrast to patterns seen in other studies. ${ }^{40} \mathrm{We}$ hypothesize that the diffuse NIRFLI pattern, which we most frequently see around dye injection sites, results from indocyanine green that is "stuck" in lymphatic capillaries and interstitial spaces, unable to move into precollectors. Our slightly different findings from those seen in Ref. 40 may result from the use of a different NIRF imaging system, different concentration of indocyanine green, different injection sites, and different types of study subjects (primary versus secondary LE, varying genetic diversity).

It is important to recognize that the volume increases in LE limbs are initially due to accumulation of stagnant lymph that drives hypertrophy and proliferation of neighboring adipose cells. ${ }^{43} \mathrm{In}$ terestingly, liposuction has been used successfully in patients with treatment-resistant LE to obtain durable limb volume reductions (some for over 20 years), but it relies on continued use of compression garments and MLD to keep lymph accumulation at bay after fat removal. ${ }^{44}$ These results suggest that, if lymph stasis is prevented (by compression garments, massage, PCT, or a combination thereof), fat re-accumulation and limb volume increases can be minimized. Consistent with this idea, a number of studies have found that intermittent pneumatic compression (IPC, another term for PCT) benefits LE patients, ${ }^{45}$ reduced LE swelling, skin fibrosis, and pain in $90 \%$ of $\mathrm{PCT}$ patients, ${ }^{46}$ improved quality of life, ${ }^{47}$ and that advanced PCT decreased total per-patient costs by $31 \%$ in 12 months post-device. ${ }^{48}$ It is possible that PCT used after lymphaticovenous anastomoses and lymph node transfer surgeries could speed recovery and improve outcomes by directing lymph-to-venous flow of anastomoses. ${ }^{49}$

The views of the lymphedematous areas during treatment, made possible by the transparent pneumatic appliances, also underscore the progressive nature of LE and the need for early intervention so that treatment may be more effective. It is likely that LE subjects with long-standing disease have accumulated subdermal adipose tissue and fibrosis that make transit of lymph occur extravascularly rather than within patent lymphatic vessels. In these cases, it is perhaps useful to imagine lymph being "squeezed" through interstitial spaces to proximal destinations (similar to water traveling through a sponge), rather than through lymphatic vessels. ${ }^{50}$ Extravascular ICG-laden lymph in Subjects 1 and 2 appeared to move from lower extremity areas to thighs and groin locales. NIRFLI 
was unable to visualize lymphatic vasculature above the ankle areas in Subject 4, who had lipolymphedema, perhaps due to interfering adipose deposition. The differences noted between Subject 4 (who was diagnosed with lipolymphedema, or LE secondary to lipedema) and Subjects 1 and 2 (with LE only) highlight the fact that LE presentations can differ depending on the initial cause of the lymphatic insufficiency.

Our results show that, in single sessions, PCT acted to enable lymph to move proximally away from edematous regions through functional lymphatic vessels when available, as in Subject 3, or otherwise through interstitial spaces/channels. Both movements were responsible for net proximal movement (and modest tissue volume reductions) in the subjects studied.

This is the first study, to our knowledge, to provide visual images of lymph movement that, in the case of Subject 1, can be compared to NIRFLI images made without PCT, and to provide visual images of lymph movement during PCT. The use of clear and windowed PCT garments enabled images that were visible in real time, provided evidence of lymph movement during PCT, and, when patent lymphatic vessels were available, provided quantitative evidence of increased lymph vessel propulsion during and after PCT. NIRFLI could potentially be used to determine which LE patients would most benefit from PCT, and to guide medical professionals in the choice of pressure and sequence settings.

\subsection{Study limitations}

This study was limited to a small number of subjects, and each subject received only one PCT treatment. Larger, longitudinal studies could determine if daily PCT enables even greater fluid removal from affected limbs and prevention of subdermal adipose and fibrotic tissue accumulation that is a hallmark symptom of LE. We did not extend imaging to time points beyond $30 \mathrm{~min}$ after the end of PCT, but it would be interesting to see how long the effects continue after treatment.

\section{Conclusions}

The results shown herein provide evidence that PCT may facilitate lymph vessel uptake and transport, as well as distal-to-proximal movement of ICG-laden lymph during a single $1 \mathrm{~h}$ treatment session in subjects with established LE who did not previously use PCT regularly. LE is a significant health burden, and complete description of the efficacy of prescribed treatments is essential for optimal health spending decisions. This report contributes information to aid understanding of PCT effectiveness in patients with various grades and types of LE.

\section{Acknowledgments}

The authors thank Eva Sevick-Muraca for helpful critique, Brenda Crews for assistance with subject recruitment and PCT therapy, Karen Ashforth for assistance with PCT therapy, and Karen Gore for assistance with subject recruitment and NIRFLI scheduling. Lympha Press USA provided pneumatic compression devices used in this study, and partial funding for personnel involved in the study. This work was partially supported by NIH/NCI grant U54 CA136404 and NIH/NCI grant R01 CA201487.

\section{References}

1. J. N. Cormier, R. L. Askew, K. S. Mungovan, Y. Xing, M. I. Ross, J. M. Armer, "Lymphedema beyond breast cancer: A systematic review and meta-analysis of cancer-related secondary lymphedema," Cancer 116, 5138-5149 (2010).

2. M. Foldi, E. Foldi, Foldi's Textbook of Lymphology, 2nd Edition, Elsevier GmbH, (2006).

3. P. Klernäs, L. J. Kristjanson, K. Johansson, "Assessment of quality of life in lymphedema patients: Validity and reliability of the Swedish version of the Lymphedema Quality of Life Inventory (LQOLI)," Lymphology 43, 135-145 (2010).

4. M. R. Fu, "Breast cancer-related lymphedema: Symptoms, diagnosis, risk reduction, and management," World J. Clin. Oncol. 5, 241-247 (2014).

5. D. M. Smeltzer, G. B. Stickler, A. Shirger, "Primary lymphedema in children and adolescents: A followup study and review," Pediatrics 76, 206-218 (1985).

6. http://rarediseases.org/rare-diseases/hereditarylymphedema/.

7. R. T. Eberhart, J. D. Raffetto, "Chronic venous insufficiency," Circulation 130, 333-346 (2014).

8. N. L. Stout Gergich, L. A. Pfalzer, C. McGarvey, B. Springer, L. H. Gerber, P. Soballe, "Preoperative assessment enables the early diagnosis and successful 
treatment of lymphedema," Cancer 112, 2809-2819 (2008).

9. C. Ketterer, "Surgical options for lymphedema following breast cancer treatment," Plast. Surg. Nurses J. 34, 82-85 (2014).

10. D. W. Chang, H. Suami, R. Skoracki, "A prospective analysis of 100 consecutive lymhovenous bypass cases for treatment of extremity lymphedema, Plast. Reconstr. Surg. 132, 1305-1314 (2013).

11. R. J. Allen Jr., M-H. Cheng, "Lymphedema surgery: Patient selection and an overview of surgical techniques," J. Surg. Oncol. 113, 923-931 (2016).

12. C. Becker, J. Assouad, M. Riquet G. Hidden, "Postmastectomy lymphedema: Long-term results following microsurgical lymph node transplantation," Ann. Surg. 243, 313-315 (2006).

13. M. Mihara, H. Hara, S. Tange, H. P. Zhou, M. Kawahara, Y. Shimizu, N. Murai, "Multisite lymphaticovenular bypass using supermicrosurgery technique for lymphedema management in lower lymphedema cases," Plast. Reconstr. Surg. 138, 262272 (2016).

14. M. F. Scaglioni, M. Arvanitakis, Y. C. Chen, P. Giovanoli, C-S. Yang, E. I. Chang, "Comprehensive review of vascularized lymph node transfers for lymphedema: Outcomes and complications," Microsurgery, Jun 7. doi: 10.1002/micr.30079 (2016) [Epub ahead of print].

15. The diagnosis and treatment of pheripheral lymphedema: 2013 consensus document of the International Society of Lymphology, Lymphology 46, 1-11 (2013).

16. I-C. Tan, E. A. Maus, J. C. Rasmussen, M. V. Marshall, K. E. Adams, C. E. Fife, L. A. Smith, W. Chan, E. M. Sevick-Muraca, "Assessment of lymphatic contractile function after manual lymphatic drainage using near-infrared fluorescence imaging," Arch. Phys. Med. Rehabil. 92, 756-764 (2011).

17. K. Ashforth, E. A. Maus, F. J. Schingale, A protocol for pneumatic compression home use, Abstract, World Congress of Lymphology, San Francisco USA, Sept. (2015).

18. Mego Afek Ac Ltd., Israel www.lympha-press.com.

19. C. J. Pappas, T. F. O'Donnell Jr. "Long-term results of compression treatment for lymphedema," J. Vasc. Surg. 16, 555-564 (1992).

20. D. M. Richmand, T. F. O'Donnell Jr, A. Zelikovski, "Sequential pneumatic compression for lymphedema-a controlled trial," Arch. Surg. 120, 1116-1119 (1985).

21. K. Johannsson, E. Lie, C. Ekdahl, J. Lindfeldt, "A randomized study comparing manual lymph drainage with sequential pneumatic compression for treatment of postoperative arm lymphedema," Lymphology 31, 56-64 (1998).

22. O. Wilburn, P. Wilburn, S. G. Rockson, "A pilot, prospective evaluation of a novel alternative for maintenance therapy of breast cancer-associated lymphedema [ISRCTN76522412]," BMC Cancer 6, 84 (2006).

23. P. Karaca-Mandic, A. T. Hirsch, S. G. Rockson, S. H. Ridner, "The cutaneous, net clinical, and health economic benefits of advanced pneumatic compression devices in patients with lymphedema," JAMA Dermatol. 151, 1187-1193 (2015).

24. M. Oremus, K. Walker, I. Dayes, P. Raina, Diagnosis and treatment of secondary lymphedema Technology Asessment Report 2010, USA Department of Health \& Human Services Agency for Healthcare Research and Quality, May 28, (2010).

25. N. J. Preston, K. Seers, P. S. Mortimer, "Physical therapies for reducing and controlling lymphedema of the limbs," Cochrane Database Syst Rev. Oct 18;(4): CD003141, (2004).

26. F. Baulieu, J. I., Baulieu, L. Vaillant, V. Secchi, J. Barsotti, "Factorial analysis in radionuclide lymphography: Assessment of the effects of sequential pneumatic compression," Lymphology 22, 178-185 (1989).

27. M. V. Marshall, J. C. Rasmussen, I-C. Tan, M. B. Aldrich, K. E. Adams, X. Wang, C. E. Fife, E. A. Maus, L. A. Smith, E. M. Sevick-Muraca, "Nearinfrared fluorescence imaging in humans with indocyanine green: A review and update," Open Surg. Oncol. J. 2, 12-25 (2010).

28. T. Yamamoto, N. Matsuda, K. Doi, A. Oshima, H. Yoshimatsu, T. Todokoro, F. Ogata, M. Mihara, M. Narushima, T. Iida, I. Koshima, "The earliest finding of indocyanine green lymhography in asymptomatic limbs of lower extremity lymphedema patients secondary to cancer treatment: The modified dermal backflow stage and concept of subclinical lymphedema," Plast. Reconstr. Surg. 128, 314e-321e (2011).

29. E. M. Sevick-Muraca, "Translation of near-infrared fluorescence imaging technologies: Emerging clinical applications," Annu. Rev. Med. 63, 217-231 (2012).

30. F. Meric-Bernstam, J. C. Rasmussen, S. Krishnamurthy, I-C. Tan, B. Zhu, J. L. Wagner, G. V. Babiera, E. A. Mittendorf, E. M. Sevick-Muraca, "Toward nodal staging of axillary lymph node basins through intradermal administration of fluorescent imaging agents," Biomed. Opt. Exp. 5, 183-196 (2013).

31. E. M. Sevick-Muraca, S. Kwon, J. C. Rasmussen, "Emerging lymphatic imaging technologies for mouse and man," J. Clin. Invest. 124, 905-914 (2014). 
32. E. M. Sevick-Muraca, R. Sharma, J. C. Rasmussen, M. V. Marshall, J. A. Wendt, H. Q. Pham, E. Bonefas, J. P. Houston, L. Sampath, K. E. Adams, D. K. Blanchard, R. E. Fisher, S. B. Chiang, R. Elledge, M. E. Mawad, "Imaging of lymph flow in breast cancer patients after microdose administration of a near-infrared fluorophore: Feasibility study," Radiology 246, 734-741 (2008).

33. P. S. Mortimer, S. G. Rockson, "New developments in the clinical aspects of lymphatic disease," J. Clin. Invest. 124, 915-921 (2014).

34. J. C. Rasmussen, I-C. Tan, M. V. Marshall, K. E. Adams, C. E. Fife, E. A. Maus, L. A. Smith, K. R. Covington, E. M. Sevick-Muraca, "Human lymphatic architecture and dynamic transport imaged using near-infrared fluorescence," Transl. Oncol. 3, 362372 (2010).

35. M. B. Aldrich, E. M. Sevick-Muraca, "Cytokines are systemic effectors of lymphatic function in acute inflammation," Cytokine 64, 362-369 (2013).

36. X. Geng, B. Cha, M. R. Mahamud, K. C. Lim, R. Silasi-Mansat, M. K. Uddin, N. Miura, L. Xia, A. M. Simon, J. D. Engel, H. Chen, F. Lupu, R. S. Srinivasan, "Multiple mouse models of primary lymphedema exhibit distinct defects in lymphovenous valve development," Dev. Biol. 409, 218-233 (2016).

37. F. Tatin, A. Taddei, A. Weston, E. Fuchs, D. Devenport, F. Tissir, T. Makinen, "Planar cell polarity protein Celsr1 regulates endothelial adherens junctions and directed cell rearrangements during valve morphogenesis," Dev. Cell. 26, 31-44 (2013).

38. M. L. Gonzalez-Garay, M. B. Aldrich, J. C. Rasmussen, R. Guilliod, P. E. Lapinski, P. D. King, E. M. Sevick-Muraca, "A novel mutation in CELSR1 is associated with hereditary lymphedema," Vasc. Cell 8, 1 (2016).

39. K. E. Adams, J. C. Rasmussen, C. Darne, I- C. Tan, M. B. Aldrich, M. V. Marshall, C. E. Fife, E. A. Maus, L. A. Smith, R. Guilliod, S. Hoy, E. M. SevickMuraca, "Direct evidence of lymphatic function improvement after advanced pneumatic compression device treatment of lymphedema," Biomed. Opt. Express. 1, 114-125 (2010).

40. T. Yamamoto, M. Narushima, K. Doi, A. Oshima, F. Ogata, M. Mihara, I. Koshima, G. S. Mundinger, "Characteristic indocyanine green lymphography findings in lower extremity lymphedema: The generation of a novel lymphedema severity staging system using dermal backflow patterns," Plast. Reconstr. Surg. 127, 1979-1986 (2011).
41. T. Yamamoto, H. Yoshimatsu, M. Narushima, N. Yamamoto, A. Hayashi, I. Koshima, "Indocyanine green lymphography findings in primary leg lymphedema," Eur. J. Vasc. Endovasc. Surg. 49, 95102 (2015).

42. T. Yamamoto, M. Narushima, H. Yoshimatsu, N. Yamamoto, A. Oka, Y. Seki, T. Todokoro, T. Iida, I. Koshima, "Indocyanine green velocity: Lymph transportation capacity deterioration with progression of lymphedema," Ann. Plast. Surg. 71, 591-594 (2013).

43. Y. Wang, G. Oliver, "Current views on the function of the lymphatic vasculature in health and disease," Genes. Dev. 24, 2115-2126 (2010).

44. H. Brorson, K. Ohlin, G. Olsson, M. K. Karlsson, "Breast cancer-related chronic arm lymphedema is associated with excess adipose and muscle tissue," Lymphat. Res. Bio. 7, 3-10 (2009).

45. A. Szuba, R. Achula, S. Rockson, "Decongestive lymphatic therapy for patients with breast carcinoma-associated lymphedema. A randomized, prospective study of a role for adjunctive intermittent pneumatic compression," Cancer 95, 22602367 (2002).

46. S. C. Muluk, A. T. Hirsch, E. C. Taffe, "Pneumatic compression device treatment of lower extremity lymphedema elicits improved limb volume and patient-reported outcomes," Eur. J. Vasc. Endovasc. Surg. 46, 480-487 (2013).

47. S. N. Blumberg, T. Berland, C. Rockman, F. Mussa, A. Brooks, N. Cayne, T. Maldonado, "Pneumatic compression improves quality of life in patients with lower extremity lymphedema," Ann. Vasc. Surg. (2015), doi: 10.1016/j.avsg.2015.07.004.

48. A. T. Hirsch, P. K. Mandic, Advanced pneumatic compression device treatment of lymphedema improves health outcomes and lowers cost, in Presented at the 11th National Lymphedema Network Int. Conf., Washington, D.C., 3-7 September (2014).

49. Y. Seki, T. Yamamoto, H. Yoshimatsu, A. Hayashi, A. Kurazono, M. Mori, Y. Kato, I. Koshima, "The superior-edge-of-the-knee incision method in lymphaticovenular anastomosis for lower extremity lymphedema," Plast. Reconstr. Surg. 136, 665e675e (2015).

50. W. L. Olszewski, P. J. Ambujam, M. Zaleska, M. Cakala, "Where do lymph and tissue fluid accumulate in lymphedema of the lower limbs caused by obliteration of lymphatic collectors?" Lymphology 42, 105-111 (2009). 